\title{
DETERMINSTIC LATERAL DISPLACEMENT MEMS DEVICE FOR CONTINUOUS BLOOD CELL SEPARATION
}

\author{
Siyang Zheng', Raylene Yung ${ }^{2}$, Yu-Chong Tail, Harvey Kasdan ${ }^{3}$ \\ ${ }^{1}$ Caltech Micromachining Lab, California Institute of Technology, USA \\ ${ }^{2}$ Stanford University, USA \\ ${ }^{3}$ Iris Diagnostics, International Remote Imaging Systems, Inc., USA
}

\begin{abstract}
This work presents a new MEMS device which uses deterministic lateral displacement for the continuous separation of leukocytes (white blood cells) and erythrocytes (red blood cells). By running blood cells in laminar flow through an array of columnar obstacles, deterministic lateral displacement asserts that the path a cell follows is determined by its size. The system consists of PDMS channels with posts on glass slides. An effective separation region of $7 \mathrm{~mm}$ by $1.8 \mathrm{~mm}$ is achieved on a $1 \mathrm{~cm}$ by $1 \mathrm{~cm}$ chip. A $420 \mu \mathrm{m}$ lateral separation was achieved for $5 \mu \mathrm{m}$ and $10 \mu \mathrm{m}$ beads. The critical particle size for separation was found experimentally to be $8 \mu \mathrm{m}$, concluded from our results using $5 \mu \mathrm{m}, 7 \mu \mathrm{m}, 8 \mu \mathrm{m}$ and $10 \mu \mathrm{m}$ polystyrene beads. Diluted whole blood and blood fraction of concentrated leukocytes were also tested with the devices in agreement with blood count results. Problem of blood cell stiction to device surfaces was investigated.
\end{abstract}

\section{INTRODUCTION}

Human blood cell separation is the first challenging step towards total blood count. Conventional cell separation methods used in blood counts almost all adopt selective cell lysis by chemicals. This unavoidably requires the reservoirs of the chemicals and makes it difficult for onchip implementation. Recently, there are a few efforts on cell separation in micro scale using, for examples, fieldflow fractionations $[1,2]$, dielectrophoretic force separation [3], magnetic separator [4] and microstep device [5]. This work aims specifically to develop a simple, reusable but deterministic separation method using mictofluidics without the need for lysing chemicals. In addition, a special attention is paid to the well-know cell stiction problem.

Human blood celis can be roughly classified into three groups: erythrocytes (RBC), leukocytes (WBC) and platelets. Leukocytes can be further classified into granulocytes, monocytes and lymphocytes. Table 1 compares the geometries and counts of erythrocytes and leukocytes in adults. Because of its shape, the height of a normal erythrocyte vaties from 1.5 to $3.5 \mu \mathrm{m}$ [6], clearly smaller than the average leukocyte whose diameter falls between 7 and $20 \mu \mathrm{m}$. This difference in size enables us to use deterministic lateral displacement to separate the two types.

The first such device was actually designed to separate submicron-sized beads or bacterial artificial chromosomes [7]. Here, we have extended the principle to design a new device for much larger particles like blood cells. In a deterministic lateral displacement device, the
Table 1: Comparison of erythrocytes and leakocyte $[6,8]$

\begin{tabular}{|l|cc|}
\hline & Erythrocyte & Leukocyte \\
\hline Cell shape & Biconcave disc & Sphere \\
Diameter average $(\mu \mathrm{m})$ & 6 & 10 \\
Diameter range $(\mu \mathrm{m})$ & $5-8$ & $7-20$ \\
Count $\left(\mathbf{1 0}^{9} / \mathrm{L}\right)$ & $4,200-5,800$ & $4.5-11,0$ \\
\hline
\end{tabular}

separation chamber consists of an array of obstacles with center-to-center distance $\lambda$, gap size $\mathrm{d}$ and shifted displacement of each row $\Delta \lambda$. The micro scale of the devices ensures laminar flow inside. Assuming uniform flow profile at gaps, the collection lane, which is where the flow bifurcates and moves around the obstacle, has a width of $\Delta \lambda$. Small particles will follow the streamlines and thus move in "zigzag mode", meaning they traveled straight through the device, only moving around obstacles in order to compensate for row shifts. Large particles with radius larger than the width of the collection lane width can not fit in, so they move in "displacement mode", meaning they traveled diagonally one way (left or right based on row shift direction) through the device, never moving around obstacles. The critical particle diameter $\mathrm{D}$, at which particles of increasing size change from zigzag to displacement, is $2 d \Delta \lambda / \lambda$. Considering non-uniform flow profile at the gaps, the critical particle diameter is:

$$
D=\eta 2 d \Delta \lambda / \lambda
$$

with coefficient $\eta$ account for the effect of non-uniform flow profile.

\section{DEVICE DESIGN AND FABRICATION}

We chose PDMS (Sylgard 184, Dow Corning, MI) for the device because of its ease of use. Devices are fabricated with DRIE-silicon molds and mounted on a glass slide by overnight baking at $80^{\circ} \mathrm{C}$ (figure 1). The channel height is $20 \mu \mathrm{m}$. The effective separation area measures $7 \mathrm{~mm}$ by $1.8 \mathrm{~mm}$ and consists of upstream and downstream regions. In each region, every consecutive row of obstacles is shifted horizontally by a fixed amount: $4 \mu \mathrm{m}$ for upstream, $6 \mu \mathrm{m}$ for downstream. In both regions, along each row, the center-to-center distance of obstacles $\lambda$ is $60 \mu \mathrm{m}$ while the distance between them $\mathrm{d}$ is $14 \mu \mathrm{m}$.

\section{DEVICE CALIBRATION}

Experimentally, the device was first calibrated with polystyrene beads. Fluids were pumped into devices with syringe pumps (Pico Plus, Harvard Apparatus, MA). Central flow rate was kept at $0.2 \mu \mathrm{l} / \mathrm{min}$ and sheath flows were $1.2 \mu \mathrm{l} / \mathrm{min}$. Bead concentrations were on the order of $10^{3} / \mu \mathrm{l}$. 


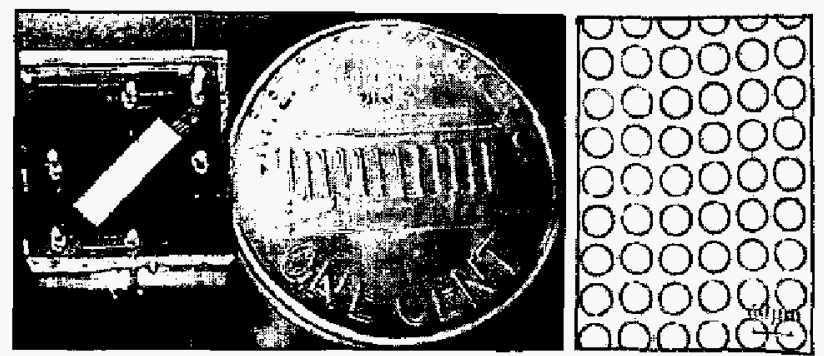

Figure 1: Device for blood separation (left) and detailed device structure (right).

All beads $5 \mu \mathrm{m}$ (green) or $7 \mu \mathrm{m}$ (red) in diameter followed the "zigzag mode". In contrast, $10 \mu \mathrm{m}$ (red) diameter beads followed the "displacement mode". Finally, the $8 \mu \mathrm{m}$ diameter (clear) beads did not strictly follow either mode and exited between the $10 \mu \mathrm{m}$ and 7 $\mu \mathrm{m}$ beads (figure 2). From a statistical analysis of a video taken near the outlet, a $420 \mu \mathrm{m}$ lateral separation was achieved for $5 \mu \mathrm{m}$ and $10 \mu \mathrm{m}$ beads (figure 3). Also from the results, the critical particle diameter $\mathrm{D}$ is around $8 \mu \mathrm{m}$.

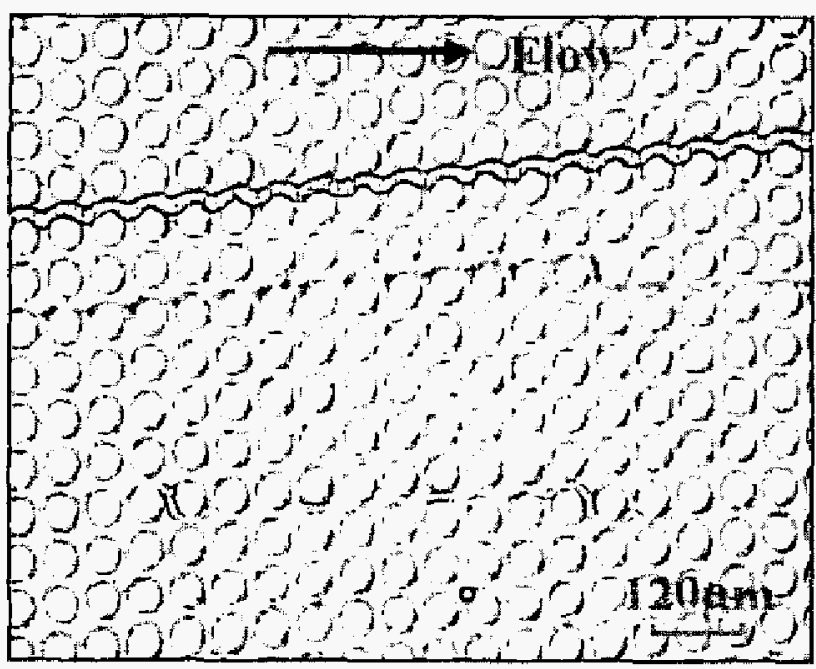

Figure 2: Separation of $5 \mu \mathrm{m}$ (green), 8 um (clear) and 10 um (red) beads. Picture was generated by overlaying of multiple video frames.

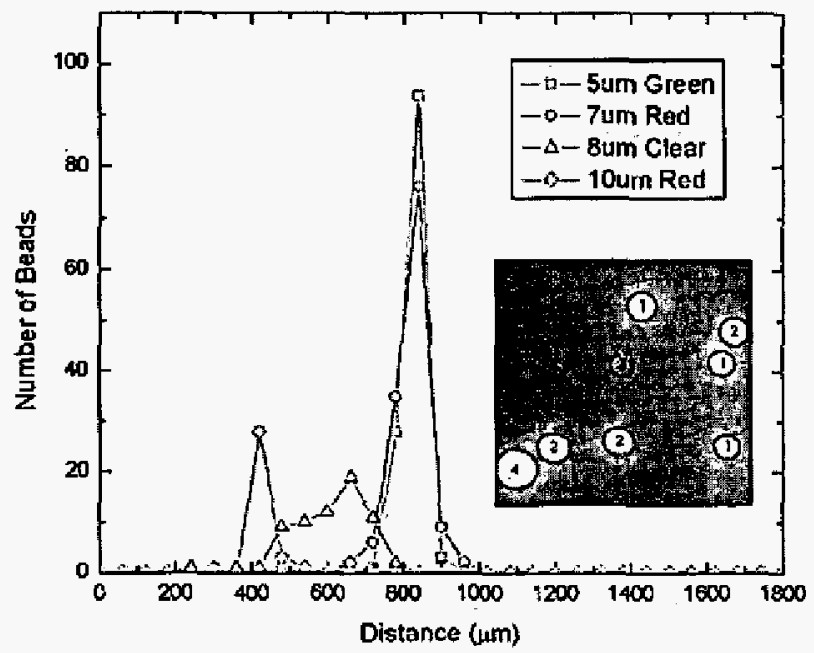

Figure 3: Statistical analysis of beads separation. Inserted picture is taken with fluorescent microscope. Beads with label 1 , 2, 3 and 4 are $5 \mu \mathrm{m}, 7 \mu \mathrm{m}, 8 \mu \mathrm{m}$ and $10 \mu \mathrm{m}$ in diameter respectively.

The effects of flow rate on separation were studied by changing flow rates followed by statistical analysis of beads separation profiles. For $5 \mu \mathrm{m}$ and $10 \mu \mathrm{m}$ beads flowing in the same device, changing central flow rates from $0.01 \mu \mathrm{l} / \mathrm{min}$ (corresponding roughly to linear velocity of $1 \mathrm{~mm} / \mathrm{sec}$ ) to $2 \mu \mathrm{l} / \mathrm{min}$ (corresponding roughly to linear velocity of $5 \mu \mathrm{m} / \mathrm{sec}$ ) shows no change on separation: 5 $\mu \mathrm{m}$ beads follow zigzag mode while $10 \mu \mathrm{m}$ beads follow displacement mode. We then flowed in $7 \mu \mathrm{m}$ fluorescent beads, which have a large size distribution (up to $16 \%$ coefficient of variation cited from manufacturer). The central flow rate was changed from $0.015 \mu 1 / \mathrm{min}$ to 1.0 $\mu \mathrm{l} / \mathrm{min}$. From figure 4 , the separation profiles remained roughly the same, with beads slightly tend to flow in displacement mode at low flow rate regimes. The size distribution of the $7 \mu \mathrm{m}$ beads needs to be compared with this result for further interpretation. Since the critical particle size for the current device is $8 \mu \mathrm{m}$, to test further flow rate effects on separation, it's reasonable to flow in 8 $\mu \mathrm{m}$ beads with different flow rates and observe possible changes on separation profiles.

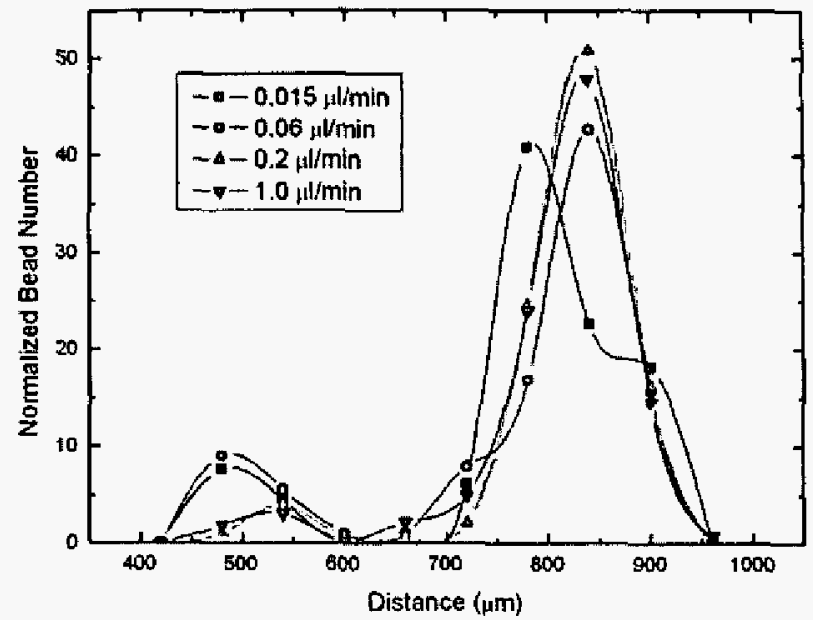

Figure 4: Separation of $7 \mu \mathrm{m}$ beads with large size distribution under different flow rates.

\section{DEVICE TESTING}

Human blood cell separation was then demonstrated using a blood fraction of concentrated leukocytes diluted with Ficoll-Paque Plus (Amersham Biosciences, Sweden). Leukocytes were concentrated by repetitive centrifugation at $1500 \mathrm{rcf}$ (relative centrifugation force, equivalent to g) for 10 minutes at $4^{\circ} \mathrm{C}$, then taking out top layer of supernatant and bottom layer of pellet. Ficoll-Paque Plus is a mixture of diatrizoate sodium and Ficoll 400. Ficoll 400 is a neutral, highly branched, hydrophilic polymer of sucrose. Traditionally it has been used as a reagent to purify lymphocytes from human peripheral blood based on density gradient centrifugation. Its density is $1.077 \pm$ $0.001 \mathrm{~g} / \mathrm{ml}$, which is more close to blood cell density than aqueous saline. Diluting blood with Ficoll-Paque Plus enables us to run experiment over one hour without severe sedimentation.

The use of a hemacytometer yielded a measured leukocyte to erythrocyte ratio of 1:43. As shown in figure 5 , two groups of cells following different flow patterns were identified. Statistical analysis concluded that the ratio of cells traveling displacement to those traveling zigzag was 1:38 (figure 6), very consistent with the leukocyte-to-erythrocyte ratio. 


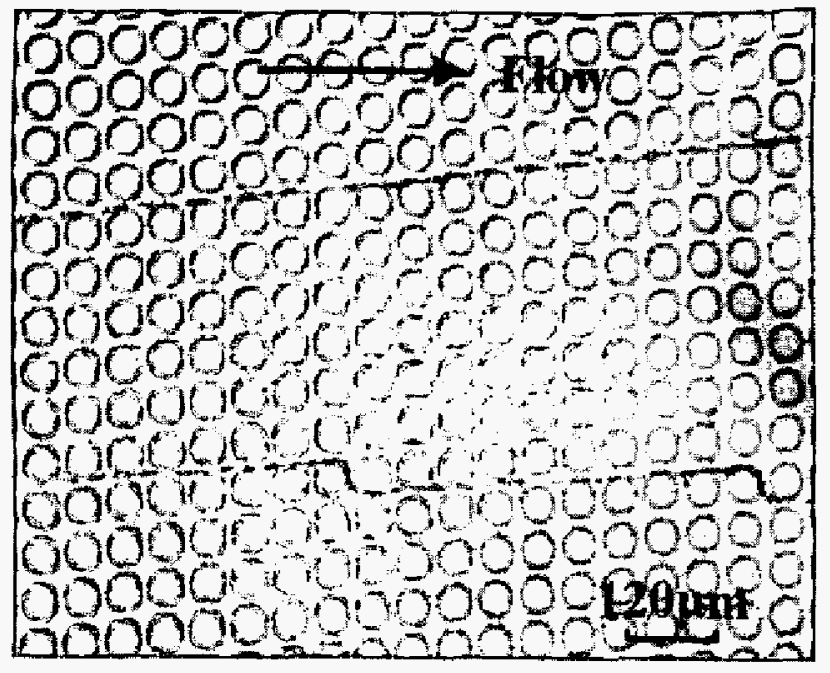

Figure 5: Separation of leukocyte (top) from erythrocyte (bottom). Picture was generated by overlaying of multiple video frames.

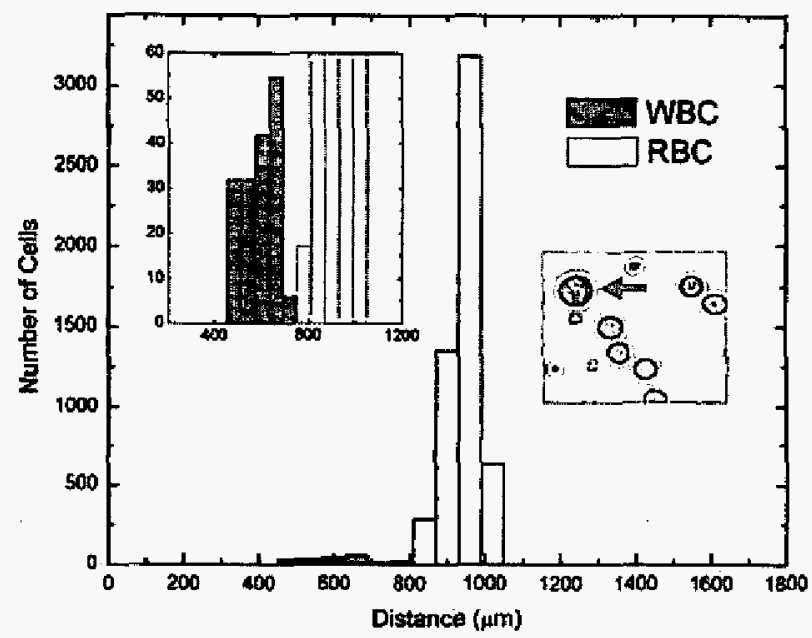

Figure 6: Statistical analysis of separation of blood fraction of concentrated leukocytes. Inserted plot (left) is a close up of lenkocyte data. Inserted plot (right) shows one leukocyte (arrow pointed) among erythrocytes.

We then performed tests using a sample of whole blood, diluted with Ficoll-Paque Plus. Because of the high erythrocyte-to-leukocyte ratio in natural blood, and limitations on flow rate and blood cell concentration, tests were run for over two hours in order to observe several leukocytes. Statistical analysis showed the ratio of cells traveling displacement to zigzag to be 1:502 (figure 7), which is in agteement with the known leukocyteerythrocyte ratio. However, towards the end of such tests, several erythrocytes flowing in zigzag mode appeared in the lanes normally occupied by cells flowing in displacement mode. Although still under investigation, we believe such phenomena are caused by the increased amount of blood cells sticking to the obstacles after a long testing time.

Cell stiction to device surface broadens cell separation profile and impair device performance. It normally happened mostly near the entrance of the blood flow (figure 8). For slight stiction or stiction in early stage as in figure 8(b), fibrous structures as well as blood cells can often be observed. Higher magnification shows

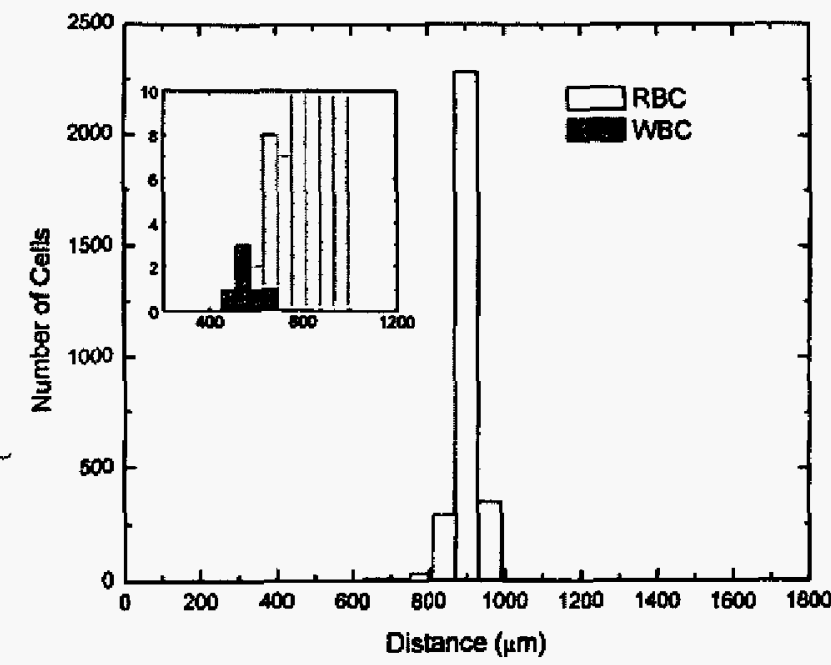

Figure 7: Statistical analysis of separation of diluted whole blood sample. Inserted plot is a close up of letukocyte data.

these fibrous structures have platelets attached. When blood is diluted over 50 times, these fibrous structures are normally observed before blood cells start to stick to obstacles. It is likely that platelets and plasma proteins involved cascaded blood coagulation happens in these situations, although the chemicals involved might be different from in vivo physiological cases. For higher concentrated blood and stiction in later stage as in figure 8(c), simple mechanical blockage as well as cascaded blood coagulation can happen.

To solve the problem of blood cell stiction to device surface, we studied the effects of dilution factor and freshness of the blood sample. The same blood sample was diluted 2, 10 and 50 times with Ficoll-Paque Plus. Experiments were performed in two consecutive days, while blood sample was kept at room temperature for all the time. In all the cases, the central flow rate was 0.08 $\mu \mathrm{l} / \mathrm{min}$ and the sheath flows were $0.4 \mu \mathrm{l} / \mathrm{min}$. Areas of blockage were measured after 30 minutes of blood flow. Roughly 24 hours after the blood was drawn, 50 times diluted sample show no sign of cell stiction. Even for 2 times diluted blood sample, the stiction is minor (figure 9). After two days, for the same dilution level, the areas of blockage increase about 15 times compared with samples of the first day. The freshness of the blood sample plays an important role in cell stiction to device surface. Normally the blood samples will be stored in refrigerator, which can greatly mitigate this effect. For experiments at the same day, the area of blockage scales roughly with the dilution factors, which can happen for both simple mechanical blockage and cascaded blood coagulation.

\section{CONCLUSIONS AND DISCUSSION}

A MEMS device was demonstrated to separate leukocytes from erythrocytes based on deterministic lateral displacement principle. Fluorescent beads of $5 \mu \mathrm{m}, 7 \mu \mathrm{m}$, $8 \mu \mathrm{m}$ and $10 \mu \mathrm{m}$ were successfully separated. The critical particle size for separation was shown to be around $8 \mu \mathrm{m}$ experimentally, which could be used for rough separation of blood cells. Ratio of blood cells following displacement mode to those in zigzag mode matches with leukocytes to erythrocytes ratio. Cell stiction to device 
surface was found to be scale linearly with blood dilution factor. Freshness of the blood sample has a significant role in cell stiction to device surface. In experiments, we observed polystyrene beads, which are more hydrophobic, were more likely to stick to device surfaces then blood cells. Even in the downstream, polystyrene beads adhere readily to obstacles. Given the hydrophilic nature of cell surfaces, the hydrophobic property of PDMS makes it better to prevent severe cell stiction.

It is also believed that in our devices, flow rate has a minor effect on separation because even at the highest flow rate $(2 \mu \mathrm{l} / \mathrm{min})$, the Reynolds number of the flow inside the device is at the order of $10^{-2}$ and the flows in our experiments are laminar. Therefore, it implies higher flow rate can be used to achieve fast separation. Using equation (1) for critical particle diameter, the coefficient $\eta$ will be between 2 to 3 to fit the data.

Future work will focus on improving device performance and incorporate sensors downstream for blood counting. A technology to specifically stain leukocytes with fluorescent dyes in Ficoll-Paque Plus system is under development. This will enable us to measure the separation efficiency of blood cells. The mechanism of separation needs more study. Simulation will be performed to help clarify flow patterns inside device, the effect of flow rate and the coefficient $\eta$. Cell stiction to devices will be further investigated, including the effects of flow rate and materials for coating device surface.

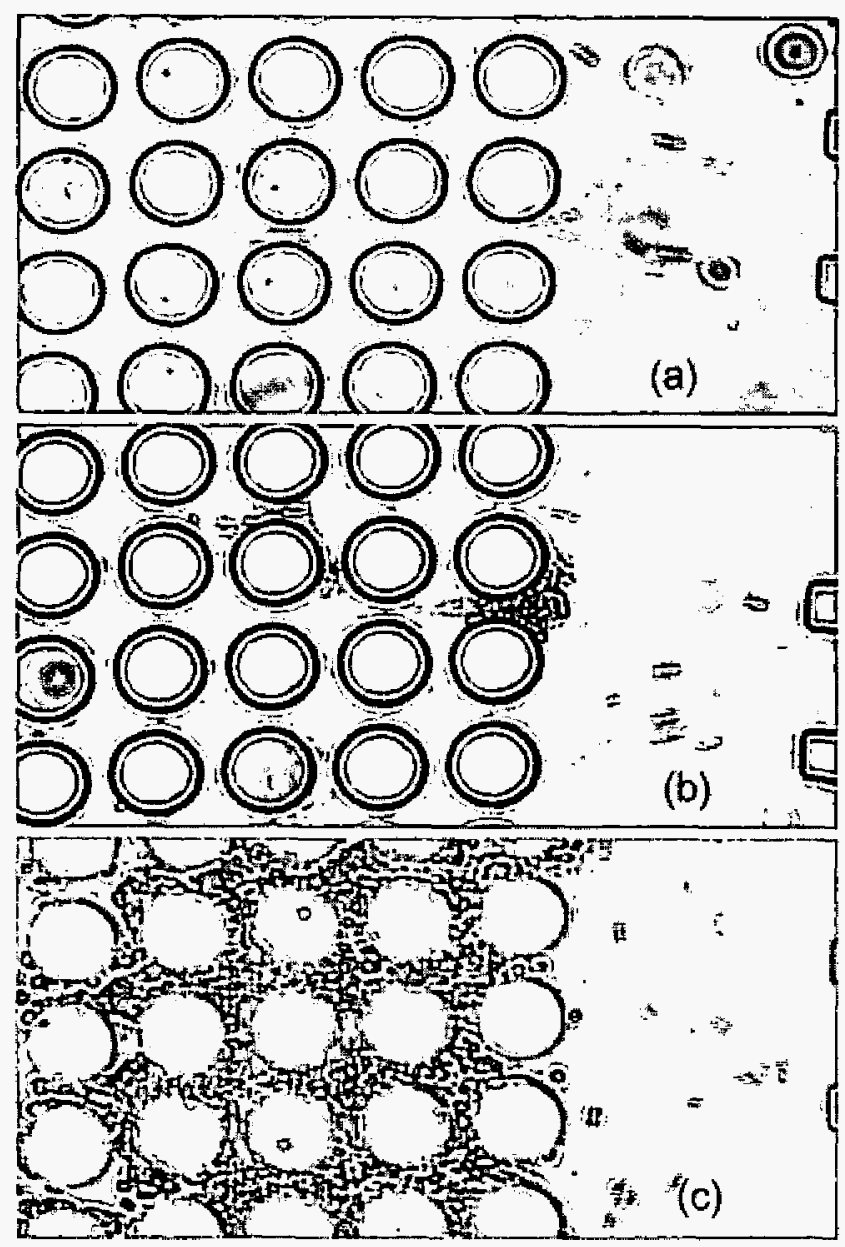

Figure 8: Different levels of stiction. (a) No obvious stiction. (b) Slight stiction. (c) Severe stiction.

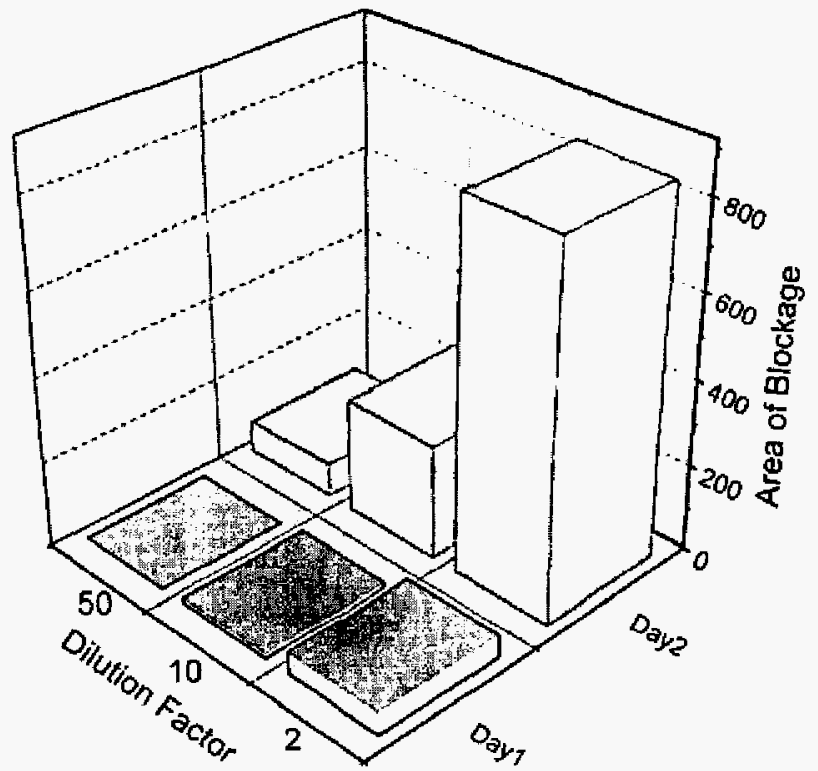

Figure 9: Effects of dilution factor and freshness of blood on blood cell stiction to device surface. Unit of area of blockage is arbitrary.

\section{ACKNOWLEDGEMENT}

This work is supported by NASA through National Space Biomedical Research Institute (NSBRI). The co-operative agreement number is NCC 9-58-317.

\section{REFERENCES}

[1] J. C. Giddings, "Field-flow Fractionation: Analysis of Macromolecular, Colloidal, and Particulate Materials," Science, vol. 260, pp. 1456-1465, 1993.

[2] J. Yang, Y. Huang, X. B. Wang, F. F. Becker, and R. C. Gascoyne, "Cell Separation on Microfabricated Electrodes Using Dielectrophoretic/gravitational Field Flow Fractionation," Analytical Chemistry, vol. 71, pp. $911-918,1999$.

[3] J. Cheng, E. L. Sheldon, L. Wu, A. Uribe, L. O. Gerrue, J. Carrino, M. J. Heller, and J. P. O'Connell, "Preparation and hybridization analysis of DNA/RNA from E-coli on microfabricated bioelectronic chips," Nature biotechnology, vol. 16, pp. 541-546, 1998.

[4] M. Berger, J. Castelino, R. Huang, M. Shah, and R. H. Austin, "Design of a Microfabricated Magnetic Cell Separator," Electrophoresis, vol, 22, pp. 3883 3892, 2001.

[5] S. Vankrunkelsven, D. Clicq, K. Pappaert, W. Ranson, C. De Tandt, H. Ottevaere, H. Thienpont, G. V. Baron, and G. Desmet, "A Novel Microstep Device for the Size Separation of Cells," Electrophoresis, vol. 25, pp. 1714-1722, 2004.

[6] H. Kasdan, "personal communication," 2004.

[7] L. R. Huang, E. C. Cox, R. H. Austin, and J. C. Sturm, "Continuous Particle Separation Through Deterministic Lateral Displacement," Science, vol. 304, pp. 987-990, 2004.

[8] S. B. McKenzie, Clinical Laboratory Hematology: Prentice Hall, 2004. 